1 A systematic evaluation of IgM and IgG antibody assay accuracy in diagnosing acute Zika Virus

2 infection in Brazil; lessons relevant to emerging infections

4 Raquel Medialdea-Carrera ${ }^{\mathrm{a}}$, Flavia Levy ${ }^{\mathrm{b}}$, Priscila Castanha ${ }^{\mathrm{c}, \mathrm{d}}$, Patricia Carvalho de Sequeira ${ }^{\mathrm{b}}$, Patricia

$5 \quad$ Brasil $^{\mathrm{e}}$, Lia L Lewis-Ximenez ${ }^{\mathrm{f}}$, Lance Turtle ${ }^{\mathrm{a}, \mathrm{g}}$, Tom Solomon $^{\mathrm{a}, \mathrm{h}}$, Ana Maria Bispo de Filippis ${ }^{\mathrm{b}}$, David

$6 \quad$ W. Brown ${ }^{\text {b,i }}$ II, Michael J. Griffiths ${ }^{\mathrm{a}, \mathrm{j}} \mathbb{I}$

$8{ }^{a}$ National Institute for Health Research (NIHR) Health Protection Research Unit in Emerging and

9 Zoonotic Infections (HPRU EZI), Institute of Infection and Global Health, University of Liverpool,

10 Liverpool, UK

$11{ }^{b}$ Flavivirus Reference Laboratory, Oswaldo Cruz Institute, Fiocruz, Rio de Janeiro, Brazil

$12{ }^{\mathrm{c}}$ Aggeu Magalhães Research Center, Oswaldo Cruz Foundation (FIOCRUZ), Recife,

13 Pernambuco, Brazil.

$14{ }^{\mathrm{d}}$ Faculty of Medical Science/ Institute of Biological Science, University of Pernambuco, Recife,

15 Pernambuco, Brazil.

$16{ }^{\mathrm{e} E v a n d r o ~ C h a g a s ~ N a t i o n a l ~ I n s t i t u t e ~ o f ~ I n f e c t i o u s ~ D i s e a s e s ; ~ O s w a l d o ~ C r u z ~ F o u n d a t i o n ~(F i o c r u z), ~ R i o ~ d e ~}$

17 Janeiro, Brazil

$18{ }^{\mathrm{f}}$ Viral Hepatitis Clinic, Viral Hepatitis National Reference Laboratory, Oswaldo Cruz Institute, Fiocruz,

19 Rio de Janeiro, Brazil 
$20{ }^{\mathrm{g}}$ Tropical \& Infectious Disease Unit, Royal Liverpool University Hospital (member of Liverpool Health

21 Partners), Liverpool, UK

$22{ }^{\mathrm{h}}$ The Walton Centre NHS Foundation Trust, Liverpool, UK

$23 \quad{ }^{\mathrm{i}}$ Public Health England, London, UK

$24 \quad{ }^{\mathrm{j}}$ Alder Hey Children's NHS Foundation Trust, Liverpool, UK

25 II These authors contributed equally to the work

26

27 RUNNING TITLE: Evaluation of Zika Virus antibody assays in Brazil

28

29 \#Address for correspondence: Department of Clinical Infection, Microbiology and Immunology, Ronald

30 Ross Building, 8 West Derby Street, Institute of Infection and Global Health, University of Liverpool,

31 L69 7BE, UK. 


\section{Abstract 249}

34 Accurate diagnostics underpin effective public health responses to emerging viruses. For viruses, such as Zika virus (ZIKV), where the viremia clears quickly, antibody-based (IgM or IgG) diagnostics are recommended for patients who present seven days after symptom onset. However, cross-reactive antibody responses can complicate test interpretation among populations where closely related viruses circulate.

We examined the accuracy (proportion of samples correctly categorized as Zika-positive or negative) for antibody-based diagnostics among Brazilian residents (Rio de Janeiro) during the ZIKV outbreak. Four ZIKV ELISAs (IgM and IgG Euroimmun, IgM Novagnost and CDC MAC), two dengue ELISAs (IgM and IgG Panbio), and the ZIKV plaque reduction neutralization test (PRNT) were evaluated. Positive samples were ZIKV PCR confirmed clinical cases collected in 2015-2016 ( $n=169)$; Negative samples $(n=236)$ were collected before ZIKV was present in Brazil $(\leq 2013)$.

Among serum samples collected $\geq 7$ days from symptom onset, PRNT exhibited the highest accuracy

The Euroimmun IgG assay, although misdiagnosing $22 \%$ of samples, provided the most accurate

53 widespread deployment, particularly where related viruses co-circulate. 


\section{Introduction}

55 Zika virus (ZIKV) is an arthropod-borne flavivirus. A public health emergency of international concern

56 (PHEIC) was declared by the World Health Organization (WHO), in response to the large Zika 57 epidemics in South and Central America in 2015-2016 (1). Although transmission has declined, over 85 countries across South and Central America, Asia, West Africa, Caribbean and the Pacific Islands have current or previous transmission of ZIKV and another 61 have stablished mosquito vectors and remain at risk for Zika infection (2-4). Moreover, autochthonous transmission of Zika Virus was demonstrated in Europe with, at least, 3 cases of vector-borne transmission in Southern France in 2019 (5). Accurate diagnostics are essential to guide appropriate clinical management of suspected patients. Both false negative and false positive diagnosis may trigger catastrophic consequences, especially among pregnant women (6).

The first-line diagnostic test for ZIKV detection is direct molecular detection using PCR. However, the time-frame for accurate virus detection following exposure using this method is short. Consequently, the WHO issued laboratory diagnostic algorithms recommending anti-ZIKV antibody-based testing in patients presenting seven or more days after symptom onset $(7,8)$. Nevertheless, accurate detection of ZIKV antibodies is challenging because antibody-based assays are susceptible to cross-reactivity from related viruses. This is a particular issue in regions such as Latin America where extensive circulation of multiple flaviviruses has occurred in the population over the last 30 years. In Brazil, all four serotypes of dengue virus (DENV) circulate and yellow fever virus (YFV) vaccination is widespread in many regions 
76 of Brazil and coverage is likely to have increased following the vaccination campaigns mounted in

77 response to recent yellow fever outbreaks $(12,13)$.

78 The WHO highlighted the important need for field validation of available Zika serological assays in

79 flavivirus exposed populations (14). A range of different antibody-based assays have been developed

$80(15,16)$, but evaluation of the assay's performance in local populations lagged behind their use.

81 To date, there are very few published studies on the performance of commercial ZIKV antibody-based

82 assays using well-characterized samples from South American populations and no systematic evaluation

83 from Brazil. Evaluations based on sera from European travelers who had visited countries with ZIKV

84 circulation found a high sensitivity and specificity for the commercial immunoglobulin M (IgM) and

85 IgG enzyme-linked immunosorbent assays (ELISAs) that use a recombinant ZIKV NS1 antigen (17-20).

86 Other commercial assays, such as the IgM $\mu$-capture ELISA, have also been approved for use in the

87 Americas, despite initial reports of low test specificity among travelers (21). The Center for Disease

88 Control and Prevention (CDC) in the USA, recommends the use of an IgM antibody capture enzyme-

89 linked immunosorbent assay (MAC-ELISA) which is licensed under the CDC FDA-emergency-use-

90 authorization protocol. However, reports from Nicaragua and the USA have shown this assay to have

91 relatively low specificity and it is no longer recommended for screening. Further local population

92 evaluations have been recommended $(22,23)$.

93 We conducted a systematic evaluation of four antibody-based methods for ZIKV diagnosis: the IgM and

94 IgG NS1 Anti-ZIKV ELISAs (Euroimmun), the IgM $\mu$-capture ZIKV ELISA (Novagnost) and the CDC

95 MAC-ELISA. We also re-evaluated two DENV antibody assays, the IgM and IgG ELISA assays

96 (Panbio) in this new context. We compared these ELISAs against the ZIKV plaque reduction

97 neutralization test (PRNT), which is currently considered the "gold standard" by the WHO for the

98 confirmatory diagnosis of Flavivirus infections. The assays were evaluated using well-characterized sera 
99 from residents of Rio de Janeiro, Brazil, classified by clinical and laboratory testing, as confirmed ZIKV 100 (cases with both clinical evidence of ZIKV infection and positive detection of ZIKV RNA by RT-PCR

101 in at least one specimen) or non-ZIKV cases (sera collected in 2013 or before, prior to the arrival of 102 ZIKV in Rio de Janeiro). Our aim was to examine assay accuracy among the Brazilian population, to 103 investigate the time window of detection of anti-ZIKV antibodies and the biological variability of 104 antibody responses.

\section{Materials and Methods}

\section{Ethics Statement}

108 The sera and patient data were used in accordance with the ethical standards of the Instituto Nacional de

109 Infectologia Evandro Chagas and the Instituto Oswaldo Cruz. The study protocol was approved by its

110 Research Ethics Committee (reference CAAE 0026.0.009.000-07 and CAAE 71405717.8.0000.5248).

111 Specimens were given laboratory numbers and so anonymized to testers to ensure patient 112 confidentiality.

\section{Study population and sample selection}

114 ELISA evaluations were performed at the Flavivirus Reference Laboratory, Institute Oswaldo Cruz in 115 Rio de Janeiro (Brazil). Plaque reduction neutralization tests (PRNT) were performed at the Aggeu 116 Magalhães Institute in Recife (Brazil).

117 Four-hundred and five serum samples in total were tested (Table 1). All sera were collected from 118 residents $(n=307)$ of the State of Rio de Janeiro. Only samples stored at $-20^{\circ} \mathrm{C}$ or below, and with no 119 history of repeated freeze-thaw were used in the study. Due to limited availability of reagents and serum 120 volumes, not all samples were tested on all assays. 
121 Panels of sera were assembled for the evaluation: 1) to assess assay sensitivity, samples from ZIKV PCR

122 confirmed cases were used (Set1: $n=169)$ from subjects $(n=71)$ with rash-fever symptom and positive

123 detection of ZIKV RNA by RT-PCR. Samples were collected in 2015 or 2016, coinciding with the peak

124 of the ZIKV epidemic in Rio de Janeiro (1). Most subjects (66 out of 71) had two or more sequential

125 samples collected (see Table S1 for further details on serum collections). 2) To assess assay specificity, 126 non-ZIKV samples, collected from individuals during, or before, 2013 were used (Set2 and Set3; $127 \mathrm{n}=236)$. These were collected 2 years before the ZIKV outbreak in Rio de Janeiro began in 2015 (24).

128 Set2 $(n=184)$ included samples from subjects with confirmed dengue (clinical presentation and PCR or 129 IgM positive; $n=90)$, measles or rubella infection $(n=40)$, who had received yellow fever vaccination $130(n=19)$, or other population samples $(n=35)$. Set3 samples were collected from patients attending a 131 hepatitis clinic at Fiocruz, Rio de Janeiro $(\mathrm{n}=52)$.

\section{Diagnostic Assays}

133 The IgM and IgG ZIKV NS1 ELISA assays (Euroimmun, Lubeck, Germany) use recombinant Zika non134 structural protein 1(NS1) as the ZIKV antigen. Assays were performed following the manufacturer's 135 instructions. IgM and IgG results were determined based on optical density (OD) ratio of human 136 sample/calibrator sample. A result was classified as: positive if ratio $\geq 1.1$; indeterminate between $\geq 0.8$ 137 and 1.1 ; negative $\leq 0.8$.

138 The Novagnost Zika Virus IgM $\mu$-capture ELISA (NovaTec Immunodiagnostica GmbH, Germany) uses 139 ZIKV NS1 antigen. Assays were conducted according to the manufacturer's instructions. Results were 140 classified based on the OD ratio of human/calibrator sample as per manufacturer's guidelines.

141 The CDC ZIKV MAC-ELISA (FDA CDC-designed IgM antibody capture ELISA) utilizes inactivated 142 whole virus antigen. The assays were performed as previously described using the US Centers for 
143 Disease Control and Prevention (CDC) emergency use authorization protocol (25). Results were based

144 on the Positive to Negative ratio $(\mathrm{P} / \mathrm{N}) . \mathrm{P}$ is obtained as the mean $\mathrm{OD}$ of the test serum, which is

145 compared with $\mathrm{N}$, the mean OD of the normal human serum/OD negative-control serum. Results were

146 reported as recommended: positive if $\mathrm{P} / \mathrm{N} \geq 3$; indeterminate if $\mathrm{P} / \mathrm{N} \geq 2$ but 3 and negative if $\mathrm{P} / \mathrm{N} 2$.

147 For the detection of DENV IgM and IgG antibodies, the commercial dengue IgG Indirect and IgM 148 Capture ELISAs (Panbio, Alere, United Kingdom) were used. Samples were considered positive for 149 previous or recent dengue infection according to the standard protocols of the manufacturer.

150 All the ELISA assays used the same volume of patient serum. All assays were stored at $2-8^{\circ} \mathrm{C}$ prior to 151 use.

152 PRNTs were performed following a published method (26), it is an adaptation of an established PRNT 153 protocol $(27,28)$. PRNT was performed using a Zika virus strain isolated in Northeast Brazil (ZIKV, 154 BR-PE243/2015). The cut-off for PRNT positivity was defined based on a 50\% reduction in plaque 155 counts $\left(\mathrm{PRNT}_{50}\right)$. ZIKV neutralizing antibody titers were estimated using a four-parameter non-linear 156 regression. Serum samples were considered positive when antibody titers were $>1: 100(\log 2)$.

159 Individuals with clinical presentation consistent with acute ZIKV infection (during 2015 and 2016) were 160 tested for detection of ZIKV nucleic acid material. RNA was extracted using the Qiamp Mini Elute 161 (Qiagen, Brazil). Reference ZIKV and DENV RT-PCR (for DENV suspected samples) were performed 162 as previously described $(29,30)$.

163 Statistical analyses and calculation of ROC Curves, sensitivity and specificity 
164 Diagnostic performance of the ELISAs was assessed by calculating accuracy (classified as true positive 165 and true negative / all cases tested); sensitivity (classified as positive / all true positives tested) and 166 specificity (classified as negative / all true negatives tested), using MedCalc Statistical Software, version 167 16.2.0 (MedCalc Software, Ostend, Belgium). Figures were generated using STATA v16 and PRISM 168 GraphPad v7 (GraphPad Software, La Jolla, CA). Samples with indeterminate or borderline results were 169 re-tested (if enough specimen was available). Samples with indeterminate results a second time were 170 considered negative.

171 This prospective cross-sectional diagnostic accuracy study is reported according to the Standards for 172 Reporting of Diagnostic Accuracy Study (STARD) (31) (Checklist S1, Flow chart S1, Flow chart S2).

\section{Results}

175 Age distribution was unimodal with a median age of 24.5 years (range $1-80 \mathrm{y}$ ). $55.4 \%$ of confirmed 176 ZIKV and non-ZIKV cases were female. Among the confirmed ZIKV samples, the median time of 177 sample collection after symptom onset was 7 days (range 1 - 276). Full details are given in Table 1.

\section{Diagnostic assays performance}

179 A detailed breakdown of accuracy, sensitivity and specificity for all assays tested are presented in Table $180 \quad 2$ and Table 3.

\section{Summary of overall accuracy}

182 Initially, we examined test accuracy among all samples, including samples collected within 7 days of 183 ZIKV symptom onset. The highest accuracy was exhibited by PRNT, followed by ZIKV IgG NS1 184 Euroimmun, MAC-ELISA, IgM NS1 Euroimmun, and IgM $\mu$-capture Novagnost ELISAs (78.8, 71.2, 
186 We also assessed the performance of the dengue assays (IgG and $\operatorname{IgM}$ ) for detecting ZIKV antibodies.

187 The DENV IgG ELISA exhibited similar results to the ZIKV IgG assays (59.7\% [181/303]) of samples

188 correctly classified). The DENV IgM ELISA demonstrated 37.2\% (122/328) samples correctly classified

189 for ZIKV.

190 The WHO recommends that samples collected up to 7 days from symptom onset are tested using a 191 ZIKV specific RT-PCR (32). Analysis was repeated excluding ZIKV samples collected less than 7 days

192 from symptom onset. The accuracy of all ZIKV assays improved. Again, the highest accuracy was 193 exhibited by PRNT followed by the IgG NS1 Euroimmun ELISA (93.7 and 77.9 respectively). The 194 ZIKV IgM (NS1 Euroimmun, $\mu$-capture Novagnost and CDC MAC) ELISAs continued to exhibit lower 195 accuracy $(73.6,72.5$ and $71.9 \%$ respectively).

196 PRNT exhibited both good sensitivity (89.8\% [79/88]) and specificity (96.1\% [98/102]). The major 197 weakness of the Euroimmun IgM and Novagnost IgM ELISAs was poor sensitivity (32.6 [29/89] and 198 48.3\% [43/89] respectively). The CDC MAC-ELISA exhibited reasonable sensitivity but poor 199 specificity (87.7\% [50/57] and 63.6\% [70/110] respectively; Tables 2 and 3). Assay sensitivity improved 200 in all the assays among samples collected $\geq 7$ to 13 days post symptom onset. Assay sensitivity decreased 201 for specimens collected $\geq 14$ days in the $\operatorname{IgM}$ and $\operatorname{IgG}$ ZIKV Euroimmun, IgM Novagnost and IgM 202 DENV assay.

\section{Comparison of anti-ZIKV IgG and IgM antibody responses}

204 We compared IgG and IgM anti-ZIKV antibody titers over time from symptom onset (measured using 205 the ZIKV NS1 Euroimmun ELISAs). Based on measuring a single sample, anti-ZIKV IgG was more 206 consistently detected than anti-ZIKV IgM among sera from confirmed ZIKV positive subjects (68\% 207 [114/168] vs. 22\% [37/167] respectively). Among paired samples, anti-ZIKV IgG more frequently 
exhibited a rise in antibody titers than $\operatorname{IgM}$ (IgG; rise 95\% [56/59]; $\geq 2$ fold 75\% [44/59]; IgM; rise $81 \%$

209 [46/57]; $\geq 2$ fold 61\% [35/57]; Figure 1). Anti-ZIKV IgG also exhibited a higher median fold increase

210 compared to IgM among paired sera (3.5 versus 2.6-fold increase [p=0.001]; taken 7 days apart

211 [median]; Figure 2). Overall, there was a more sustained rise in anti-ZIKV IgG compared to IgM over

212 time from symptom onset (Figure 3).

\section{Anti-DENV IgM and IgG responses in confirmed ZIKV cases}

214 Both IgM and IgG anti-DENV ELISAs gave positive results among sera collected $\geq 7$ days from PCR 215 confirmed ZIKV cases (41\% [37/90] and 88\% [79/90] respectively - for dengue IgM these are likely to 216 represent false positive IgM results) (Table 3). In an attempt to distinguish between the dengue IgG 217 response in ZIKV patients who had previously been exposed to DENV and acute ZIKV patients 218 exhibiting a false positive cross-reaction against DENV IgG antibodies, we looked at sera collected $<7$ 219 days from symptom onset. A high proportion of confirmed ZIKV cases were DENV IgG positive $(83 \%$ 220 [65/78]). A significant correlation between anti-ZIKV and anti-DENV antibody titers were observed 221 when the same sera were measured by IgG NS1 Euroimmun ZIKV and IgG Panbio DENV assays (IgG $222 r^{2}=0.258 ; p<0.0001 ;$ Figure 4a). A similar, but less significant correlation, was observed between ZIKV 223 Euroimmun IgM NS1 and Panbio DENV IgM assays $\left(\operatorname{IgM} r^{2}=0.015 ; p=0.03\right.$; Figure 4b).

\section{Diversity of anti-ZIKV IgM antibody responses}

225 To describe the variation in duration and magnitude of anti-ZIKV antibody responses, we compared 226 Zika antibody patterns in four PCR confirmed ZIKV patients (A-D, who had sera collected at $\geq 5$ 227 different time-points after symptom onset (range: 0-276 days). Antibody titers were measured by the 228 IgM NS1 and $\mu$-capture assays (Figure 5). Antibody patterns were diverse in both magnitude and 229 duration of response. Interestingly, in patient A, despite an initial rise in IgM titers, this quickly fell. IgM 
230 was below the Euroimmun NS1 ZIKV assay's positive threshold by day 22. We then looked at all

231 samples from confirmed ZIKV subjects. At 14, 27 and 90 days post symptom onset only 28.6\% (14/49),

$2328 \%(4 / 27)$, and $0 \%(0 / 14)$ samples had detectible IgM responses when measured in the ZIKV

233 Euroimmun NS1 IgM assay. Similarly, only 44.9\% (22/49), 25.9\% (7/27) and 7.1\% (1/14) samples were

234 positive, at 14, 27 and 90 days, when the same samples were measured using the $\operatorname{IgM} \mu$-capture

235 Novagnost assay.

\section{Improving the accuracy of the IgG NS1 ELISA}

237 The accuracy of the best performing ZIKV ELISA (i.e. the Euroimmun IgG NS1 ELISA) could be

238 improved by modifying the cut-off used to classify a positive result. The modified cut-off was identified

239 using receiver operator curve (ROC) analyses, defining the point at which the cut-off gave the highest

240 likelihood ratio (33) using all sera from non-ZIKV subjects ( $\mathrm{n}=204)$ and sera collected $\geq 7$ days among

241 confirmed ZIKV subjects $(\mathrm{n}=90)$. Using a cut-off of 1.5 , which provided the maximum likelihood ratio

242 (>4.4), the ELISA exhibited an accuracy of $81.0 \%$ (previously $77.9 \%$ ). Sensitivity and specificity were

24378.9 and $82.2 \%$ respectively (Figure 6; Table S2).

\section{Discussion}

246 ZIKV was a viral infection of significant international public health concern that affected over 148 247 countries during 2015-2019 (5, 34). Pregnant women are still advised not to travel in Brazil and other 248 South American countries. Among people with suspected ZIKV presenting seven or more days from 249 symptom onset serological antibody testing remains the recommended diagnostic approach (32). The 250 majority of ZIKV antibody tests employed in the field have not been validated in their target 251 populations. Despite the ZIKV outbreak in Brazil triggering WHO to declare a public health emergency, 
252 there has been no systematic evaluation of the commercial ZIKV antibody assays among Brazilians 253 residents.

254 The Euroimmun IgG NS1 assay gave the most accurate diagnostic performance among the ELISAs 255 tested. Accuracy could be improved to $81 \%$ by modifying the cut-off (from that suggested by the 256 manufacturer). Our results indicate that approximately 1 in 5 subjects are falsely classified by the 257 Euroimmun IgG ELISA when testing a single serum sample. One accepted weakness of employing an 258 IgG based ELISA is that a positive result from a single sample does not discriminate recent from past 259 infection. Akin to other IgG based tests used to diagnose acute infection (35), one option for improving 260 the sensitivity of acute ZIKV diagnosis may be to collect and test serial (paired) samples. During testing 261 of ZIKV PCR positive cases, $95 \%$ of paired sera exhibited a rise in antibody levels when measured via 262 the Euroimmun NS1 IgG ELISA (collected seven days apart).

263 All of the anti-ZIKV IgM ELISAs (Euroimmun NS1, $\mu$-capture and MAC) exhibited lower accuracy $264(<75 \%)$. The ELISAs tended to exhibit low sensitivity for detecting PCR confirmed ZIKV cases, even 265 when serum was collected $\geq 7$ days post symptom onset.

266 We did not expect the Euroimmun IgG ELISA to give higher sensitivity than the IgM based ELISAs. 267 Over time from symptom onset, the Euroimmun IgG ELISA exhibited more consistent and sustained 268 detection of anti-ZIKV antibody compared to its counter-part anti-ZIKV IgM ELISA. These patterns of 269 IgG and IgM response suggest a secondary immune response to infection (Figures 3 and 7), with an 270 anamnestic boosting of prior immune response most likely due to dengue that cross-reacts in the anti271 ZIKV IgG antibody assay (Figure 7). Given our patients presented with their first reported ZIKV 272 infection, their antibody responses suggest they had previously been exposed to a similar virus (i.e. to 273 dengue or another flavivirus). We suggest the prominent IgG response and poor specificity of the anti274 Zika IgG assays observed in this study reflect an anamnestic cross-reactive IgG antibody response 
275 among local Brazilians who have previously been exposed to other flaviviruses. This warrants further

276 investigation and has implications in the design of both future diagnostic tests and vaccines against

277 ZIKV and DENV in flavivirus exposed populations as it is recognized with dengue.

278 Our findings contrast markedly with published studies conducted using sera from travelers. Such studies

279 largely tested people visiting but not living in ZIKV or other flavivirus exposed countries. These latter 280 studies reported much higher sensitivity (>90\%) for the commercial IgM assays $(17,18,20)$ and higher 281 specificity for the IgG NS1 assay (>90\%) (21). The CDC MAC-ELISA exhibited reduced accuracy and 282 sensitivity when tested among Nicaraguan and Colombian residents compared to "traveler" subjects. 283 Our findings were consistent with this $[16,17]$.

284 This disparity is likely to reflect the different flavivirus exposure between visitors and local residents. 285 ZIKV positive visitors are likely to experience their first exposure to flavivirus infection. In contrast to 286 residents of Brazil, who are likely to have been exposed to ZIKV and other circulating flaviviruses in the 287 past (e.g. DENV and/or YFV). If past flavivirus exposure triggers an anamnestic antibody response 288 leading to more IgG than IgM production, this could, in part, explain the poor sensitivity of IgM based 289 ZIKV ELISAs seen here, as it has been shown previously for other flaviviruses (36). Further PRNT 290 testing specific for DENV and YFV could be conducted to ascertain prior flavivirus exposure in the 291 specimens evaluated. Our findings indicate that validation of diagnostic assays should be performed in 292 the population it will be used for.

293 The high rate of DENV IgM assay positivity (43\%) among confirmed ZIKV cases was not anticipated 294 (37). The significant correlation between antibody titers for DENV and ZIKV, when tested via IgG or 295 IgM based ELISAs, indicate there is a degree of cross-reaction both ways following DENV and ZIKV 296 infection detected in these ELISAs. These findings highlight the diagnostic challenges ahead as 297 outbreaks of both DENV and ZIKV have been forecast to re-occur in overlapping geographical regions. 
Our study findings for the ZIKV diagnostic antibody tests are pertinent to all emerging epidemics,

299 including the current SARS-CoV-2 pandemic. Our results highlight that confirming the accuracy of a

300 diagnostic assay in the target population is imperative to control and manage false positive or negative

301 results across different settings. Such validation should be advocated by governments, national public

302 health agencies and the WHO prior to test deployment. Our results also highlight the need to re-evaluate

303 the accuracy of established tests when a closely-related emergent pathogen is introduced in a region or

304 the population changes. In the case of ZIKV, we recommend the re-evaluation of DENV and YFV

assays' performance in Brazil. However, for newer threats, such as SARS-CoV-2, re-evaluation of

306 Severe Acute Respiratory Syndrome (SARS) coronavirus antibody tests should be considered among

307 Saudi Arabians and other populations with a history of transmission for closely related viruses.

308 Based on the assays we have assessed, ZIKV PRNTs provide the most accurate assay to diagnose

309 previous exposure to ZIKV among Brazilian residents in samples collected $\geq 7$ days post symptom onset.

310 Performing PRNTs requires specialized training, sophisticated laboratories and the assays are labor

311 intensive; they are therefore unlikely to be widely used for diagnosis outside of reference laboratories

312 (38). As recommended by the WHO, we support their use as a 'gold standard' reference test for

313 flavivirus diagnosis, including ZIKV, if used with an appropriate cutoff to exclude low level cross-

314 reactions (8).

315 The Euroimmun IgG NS1 ELISA provided the most accurate ELISA test to diagnose previous exposure 316 to ZIKV among Brazilian residents in samples collected $\geq 7$ days post symptom onset. In order to assess 317 whether exposure is acute, we would recommend taking paired samples (7 days apart) and looking for a 318 rise in antibody titers. The best time of collecting for these samples has not been systematically assessed 319 in this study. Based on our observational data, samples collected on days 2 and 9 post symptom onset 320 were associated with the highest fold-changes. However, this was assessed during the first waves of 
321 infection with a newly introduced virus and once significant population exposure has occurred

322 interpreting the significance of a positive Zika IgG for acute diagnosis will be even more challenging.

323 In our study, the ZIKV IgM based ELISAs exhibited poor accuracy (Euroimmun, Novagnost and MAC-

324 ELISA). Similarly, the Panbio DENV ELISAs (particularly IgM) relatively high positivity rate among

325 acute ZIKV cases was a concern. DENV antibody-based serological assays continue to be needed to

326 complement dengue PCR testing. Our findings highlight the need for careful interpretation of existing

327 dengue ELISA results. As more accurate tests are developed, their accuracy in PCR confirmed ZIKV

328 and DENV exposed residents should be comprehensively assessed.

329 Our study has limitations. Serial samples among ZIKV PCR positive subjects were collected non-

330 systematically as convenience samples. Consequently, we cannot confirm the best time to collect paired

331 samples post symptom onset. Similarly, serial samples were not available for our non-ZIKV subjects, so

332 we were unable to assess the specificity of paired samples testing in the Euroimmun NS1 IgG ELISA.

333 There remains potential for a false positive rise in titers in acutely ill non-ZIKV subjects due to antibody

334 cross-reaction. The ZIKV PCR positive subjects were not tested for DENV by PCR, so we are unable to

335 confirm that the positive DENV IgM results are false. Nevertheless, limited dengue circulation was

336 reported during the study period in the Rio de Janeiro region. Further work is needed.

337 In conclusion, this is a systematic evaluation of antibody-based ZIKV assays in Brazil. Among ZIKV

338 patients, anti-ZIKV IgG was detected more consistently than IgM, suggesting a secondary antibody

339 response to infection. ZIKV PRNT exhibited the highest accuracy of all assays tested if used with an

340 appropriate cut-off. All ZIKV IgM based ELISAs exhibited low accuracy. The Euroimmun NS1 IgG

341 ELISA exhibited the best ELISA accuracy. Nevertheless, when testing a single serum sample, it

342 misdiagnosed 1 in 5 cases. Testing paired samples via ZIKV IgG based ELISA, may offer a more

343 sensitive method of diagnosing acute ZIKV exposure. Our findings highlight that diagnostic antibody 
344 assay use and interpretation needs careful assessment in the target population, particularly when

345 deployed among populations exposed to multiple closely related viruses.

\section{Acknowledgements}

349 The authors want to thank especially the Flavivirus Laboratory, IOC, Fiocruz including Rita Maria 350 Nogueira, Elaine de Araujo, Simone Sampaio, Carolina Nogueira dos Santos, Celeste, Cintia, Marcus

351 etc. The authors want to thank the Influenza laboratory, IOC, Fiocruz including Marilda Sequeira and 352 the Viral Hepatitis Laboratory including Juliana Gil Melgaço and Paulo Sergio Fonseca de Sousa. RMC 353 thanks the award of the James Porterfield Prize in International Virology.

354 This work was supported by the United Kingdom Medical Research Council (https://www.mrc.ac.uk/, 355 Grant number MC_PC_15101); the National Institute for Health Research Health Protection Research 356 Unit (NIHR HPRU, http://www.hpruezi.nihr.ac.uk/) in Emerging and Zoonotic Infections at the 357 University of Liverpool, in partnership with Public Health England (PHE) and Liverpool School of 358 Tropical Medicine (LSTM); and the European Union's Horizon 2020 research and innovation program 359 ZikaPlan under grant agreement No. 734584 (https://ec.europa.eu/programmes/horizon2020/). The 360 views expressed are those of the authors and not necessarily those of the NHS, the NIHR, the 361 Department of Health or Public Health England. The funders had no role in study design, data collection 362 and analysis, decision to publish, or preparation of the manuscript. D B. is partially funded by the 363 European Union's horizon 2020 research and innovation program (grant agreement no. 734857), 364 ZikAction. P.B. is partially funded by CNPQ 307282/2017-1 and FAPERJ E-26/202.862/2018. LT is 365 supported by the Wellcome Trust (grant number 205228/Z/16/Z), 
366 Declaration of interests: The authors have declared that no competing interests exist.

\section{References}

369 1. Kindhauser MK, Allen T, Frank V, Santhana RS, Dye C. 2016. Zika: the origin and spread of a 370 mosquito-borne virus. Bull World Health Organ 94:675-686C.

371 2. Centers for Disease Control and Prevention C. 2018. Zika Travel Information. Traveler's Health $372 \quad$ CDC.

373 3. World Health Organization (WHO). 2019. Zika Epidemiology Update. Zika Virus Disease.

374 4. (WHO); WHO. 2019. Zika Epidemiology Update. Geneva.

375 5. Sandra Giron FF, Anne Decoppet, Bernard Cadiou, Thierry Travaglini, Laurence Thirion,

384 7. (CDC) CfDCaP. 2017. Guidance for US Laboratories Testing for Zika Virus Infection, July 24, 2017.

386 8. World Health Organization (WHO). 2016. Laboratory testing for Zika virus infection. Interim guidance World Health Organization. Interim Guidance. 
388 9. Teixeira MCM, C; Barreto, F; Barreto, ML. 2009. Dengue: twenty-five years since reemergence 389 in Brazil. Cad Saude Publica 25

390 10. Argolo AF, Feres VC, Silveira LA, Oliveira AC, Pereira LA, Junior JB, Braga C, Martelli CM. 2013. Prevalence and incidence of dengue virus and antibody placental transfer during late

11. Chiaravalloti-Neto F, da Silva RA, Zini N, da Silva GCD, da Silva NS, Parra MCP, Dibo MR, Estofolete CF, Favaro EA, Dutra KR, Mota MTO, Guimaraes GF, Terzian ACB, Blangiardo M, Nogueira ML. 2019. Seroprevalence for dengue virus in a hyperendemic area and associated socioeconomic and demographic factors using a cross-sectional design and a geostatistical

13. Organization. WH. 2018. Yellow fever-Brazil. Disease outbreak news. Emergency preparecness response Report. . Geneva, Switzerland: World Health Organization;.

14. Charrel RL-G, I; Pas, S; Lamballerie, X; Koopmans, M. \& Reusken, C. . 2016. Background Review for diagnostic test development for Zika virus infection. Bulletin of the World Health Organization 94:574.

16. Michelson Y, Lustig Y, Avivi S, Schwartz E, Danielli A. 2019. Highly Sensitive and Specific Zika Virus Serological Assays Using a Magnetic Modulation Biosensing System. J Infect Dis 219:1035-1043.

15. Murtagh MM. 2017. Zika Virus Infection Diagnostics Landscape. The International Diagnostics

\section{Centre}

17. Huzly D, Hanselmann I, Schmidt-Chanasit J, Panning M. 2016. High specificity of a novel Zika virus ELISA in European patients after exposure to different flaviviruses. Euro Surveill 21. 
412 18. Steinhagen K, Probst C, Radzimski C, Schmidt-Chanasit J, Emmerich P, van Esbroeck M, Schinkel J, Grobusch MP, Goorhuis A, Warnecke JM, Lattwein E, Komorowski L, Deerberg A,

19. Van Esbroeck M, Meersman K, Michiels J, Arien KK, Van den Bossche D. 2016. Letter to the editor: Specificity of Zika virus ELISA: interference with malaria. Euro Surveill 21.

20. L'Huillier AG, Hamid-Allie A, Kristjanson E, Papageorgiou L, Hung S, Wong CF, Stein DR,

21. Safronetz D, Sloan A, Stein DR, Mendoza E, Barairo N, Ranadheera C, Scharikow L, Holloway

K, Robinson A, Traykova-Andonova M, Makowski K, Dimitrova K, Giles E, Hiebert J, Mogk R, Beddome S, Drebot M. 2017. Evaluation of 5 Commercially Available Zika Virus Immunoassays. Emerg Infect Dis 23:1577-1580.

23. Granger D, Hilgart H, Misner L, Christensen J, Bistodeau S, Palm J, Strain AK, Konstantinovski 
435 24. Brasil P, Calvet GA, Siqueira AM, Wakimoto M, de Sequeira PC, Nobre A, Quintana Mde S,

436 Mendonca MC, Lupi O, de Souza RV, Romero C, Zogbi H, Bressan Cda S, Alves SS, Lourencode-Oliveira R, Nogueira RM, Carvalho MS, de Filippis AM, Jaenisch T. 2016. Zika Virus

25. Centers for Disease Control and Prevention C. 2016. Zika MAC-ELISA: For Use Under an Emergency Use Authorization Only - Instructions for Use.

442 26. Russell PK, Nisalak A, Sukhavachana P, Vivona S. 1967. A plaque reduction test for dengue $443 \quad$ virus neutralizing antibodies. J Immunol 99:285-90.

444 27. Castanha PM, Cordeiro MT, Martelli CM, Souza WV, Marques ET, Jr., Braga C. 2013. Force of infection of dengue serotypes in a population-based study in the northeast of Brazil. Epidemiol

28. Castanha PMS, Souza WV, Braga C, Araujo TVB, Ximenes RAA, Albuquerque M, Montarroyos UR, Miranda-Filho DB, Cordeiro MT, Dhalia R, Marques ETA, Jr., Rodrigues LC,

29. Lanciotti RS, Kosoy OL, Laven JJ, Velez JO, Lambert AJ, Johnson AJ, Stanfield SM, Duffy

30. Santiago GA, Vergne E, Quiles Y, Cosme J, Vazquez J, Medina JF, Medina F, Colon C, 
458 31. Bossuyt PM, Reitsma JB, Bruns DE, Gatsonis CA, Glasziou PP, Irwig L, Lijmer JG, Moher D,

Rennie D, de Vet HC, Kressel HY, Rifai N, Golub RM, Altman DG, Hooft L, Korevaar DA,

Cohen JF, Group S. 2015. STARD 2015: an updated list of essential items for reporting

32. World Health Organization. 2016. Laboratory testing for Zika virus infection. Interim guidance 23 March 2016.

http://apps.who.int/iris/bitstream/10665/204671/1/WHO_ZIKV_LAB_16.1_eng.pdf?ua=1.

33. Hajian-Tilaki K. 2013. Receiver Operating Characteristic (ROC) Curve Analysis for Medical

Diagnostic Test Evaluation. Caspian J Intern Med 4:627-35.

34. Organization WH. 2018. Zika virus (ZIKV) classification table data as of 15 February 2018 [Internet]. Geneva; World Health Organization.

35. Hang VT, Nguyet NM, Trung DT, Tricou V, Yoksan S, Dung NM, Van Ngoc T, Hien TT, Farrar J, Wills B, Simmons CP. 2009. Diagnostic accuracy of NS1 ELISA and lateral flow rapid tests for dengue sensitivity, specificity and relationship to viraemia and antibody responses. PLoS

36. Solomon T, Thao LT, Dung NM, Kneen R, Hung NT, Nisalak A, Vaughn DW, Farrar J, Hien immunoglobulin M dot enzyme immunoassay. J Clin Microbiol 36:2030-4. 
481 38. Thomas SJ, Nisalak A, Anderson KB, Libraty DH, Kalayanarooj S, Vaughn DW, Putnak R, 482 Gibbons RV, Jarman R, Endy TP. 2009. Dengue plaque reduction neutralization test (PRNT) in 483 primary and secondary dengue virus infections: How alterations in assay conditions impact 484 performance. Am J Trop Med Hyg 81:825-33. 


\section{Tables and Figures:}

487 Table 1 Characteristics of the study population

\begin{tabular}{|c|c|c|c|c|c|}
\hline & & No. of patients & No. of samples & $\%$ of patients & Year. \\
\hline Total & & 307 & 405 & - & $2002-2016$ \\
\hline Sex & Female & $164(296)^{*}$ & - & $55.4 \%$ & - \\
\hline \multicolumn{6}{|l|}{ ZIKV Positive samples (Set1) } \\
\hline & Total & 71 & 169 & $23.1(71 / 307)^{\mathrm{a}}$ & 2015-2016 \\
\hline & 1 Serum & 5 & 5 & $7.0(5 / 71)^{\mathrm{b}}$ & 2015-2016 \\
\hline & 2 Serum & 55 & 110 & $77.5(55 / 71)^{\mathrm{b}}$ & $2015-2016$ \\
\hline & 3 or more samples & 11 & 54 & $15.5(11 / 71)^{b}$ & 2015-2016 \\
\hline \multicolumn{6}{|l|}{ Controls - ZIKV Negative (Set2) } \\
\hline & Total & 184 & 184 & $59.9(184 / 307)^{a}$ & 2002-2013 \\
\hline & DENV1-4 (Total) & 90 & 90 & $82.6(90 / 109)^{d}$ & $2002-2013$ \\
\hline & DENV 1 & 21 & 21 & $23.3(21 / 90)^{\mathrm{c}}$ & 2010-2011 \\
\hline \multirow[t]{3}{*}{ DENV } & DENV 2 & 17 & 17 & $18.9(17 / 90)^{\mathrm{c}}$ & $2008,2010,2011$ \\
\hline & DENV 3 & 21 & 21 & $23.3(21 / 90)^{\mathrm{c}}$ & $2002,2007,2008$ \\
\hline & DENV 4 & 31 & 31 & $34.4(31 / 90)^{\mathrm{c}}$ & 2012,2013 \\
\hline Yellow fever & & 19 & 19 & $17.4(19 / 109)^{\mathrm{d}}$ & 2003-2007 \\
\hline Measles or Rubella & & 40 & 40 & $21.7(40 / 184)^{\mathrm{e}}$ & 2011-2012 \\
\hline Other non-flavivirus infections & & 35 & 35 & $19.0(35 / 184)^{\mathrm{e}}$ & 2011-2012 \\
\hline General population (Set3) & Total Set3 & 52 & 52 & $16.9(52 / 307) \mathrm{a}$ & 2013 \\
\hline
\end{tabular}

488 Abbreviations: No., number, Year, Year of sample collection, *Sex was not documented for 18 patients.

489 The population data for the subgroups denoted by roman superscript letters a through e add up to $100 \%$ :

$490{ }^{\mathrm{a}}$. represents patients in Set1, 2 and $3 .^{\mathrm{b}}$. Patients in Set1. ${ }^{\mathrm{c}}$. Dengue Positive patients. ${ }^{\mathrm{d}}$. Flavivirus

491 positive subjects. ${ }^{\mathrm{e}}$. Non-flavivirus infections subjects. 
Table 2 Sensitivity and specificity for the four anti-ZIKV antibody ELISAs

\begin{tabular}{|c|c|c|c|c|c|c|c|c|c|c|c|c|}
\hline \multirow[b]{3}{*}{ Zika Positive } & \multicolumn{3}{|c|}{ IgM Euroimmun NS1 ELISA } & \multicolumn{3}{|c|}{ IgG Euroimmun NS1 ELISA } & \multicolumn{3}{|c|}{ CDC Zika MAC-ELISA } & \multicolumn{3}{|c|}{ IgM $\mu$-capture Novagnost } \\
\hline & $\begin{array}{c}\text { Tested } \\
\text { (n) }\end{array}$ & $\%$ Sens. $(95 \% \mathrm{CI})$ & $\%$ Spec. $(95 \%$ CI $)$ & $\begin{array}{c}\text { Te } \\
\text { ste } \\
\text { d }\end{array}$ & $\%$ Sens. $(95 \%$ CI $)$ & $\begin{array}{l}\text { \% Spec. }(95 \% \\
\text { CI) }\end{array}$ & $\begin{array}{l}\text { Tes } \\
\text { ted } \\
(\mathbf{n})\end{array}$ & $\begin{array}{l}\% \text { Sens. } \\
\text { (95\% CI) }\end{array}$ & $\begin{array}{c}\% \text { Spec. }(95 \% \\
\text { CI) }\end{array}$ & $\begin{array}{c}\text { Tested } \\
(\mathbf{n})\end{array}$ & $\%$ Sens. $(95 \% \mathrm{CI})$ & \% Spec. $(95 \%$ CI) \\
\hline & 167 & $22.2(15.8-28.4)$ & & $\begin{array}{c}16 \\
8\end{array}$ & $67.8(60.8-74.9)$ & & 125 & $62.4(53.9-70.9)$ & & 167 & $31.7(24.7-38.8)$ & \\
\hline Zika ( 1-6 Days) & 78 & $10.3(3.5-16.9)$ & & 78 & $46.2(39.1-53.2)$ & & 68 & $41.2(32.7-49.7)$ & & 78 & $12.8(5.8-19.9)$ & \\
\hline Zika ( $\geq 7-13$ Days) & 40 & $37.5(22.5-52.5)$ & & 41 & $80.5(73.4-87.5)$ & & 34 & $85.3(76.8-93.8)$ & & 40 & $52.5(45.4-59.6)$ & \\
\hline Zika ( $\geq 14$ Days) & 49 & $28.5(15.9-41.2)$ & & 49 & $91.8(84.7-98.9)$ & & 23 & $91.3(82.8-99.8)$ & & 49 & $44.9(37.8-52)$ & \\
\hline Zika ( $\geq 7$ Days) & 89 & $32.6(22.9-42.3)$ & & 90 & $86.7(79.6-93.7)$ & & 57 & $87.7(79.2-96.2)$ & & 89 & $48.3(41.3-55.4)$ & \\
\hline $\begin{array}{l}\text { Non ZIKV (Flavivirus } \\
\text { and Non-flavivirus) }\end{array}$ & 166 & & $96.3(93.5-99.2)$ & $\begin{array}{c}20 \\
4\end{array}$ & & 74.0(67.4-79.9) & 110 & & $63.6(54.7-72.6)$ & 110 & & $92.7(87.9-97.6)$ \\
\hline DENV (all) & 89 & & $100.0(100.0-100.0)$ & 89 & & $76.4(67.6-85.2)$ & 88 & & $61.3(51.2-71.5)$ & 79 & & $92.4(86.6-98.25)$ \\
\hline DENV1 & 21 & & $100.0(100.0-100.0)$ & 21 & & $95.2(86.1-100.0)$ & 21 & & $52.3(31.0-73.7)$ & 16 & & $100.0(100.0-100.0)$ \\
\hline DENV2 & 17 & & $100.0(100.0-100.0)$ & 17 & & $76.4(56.3-96.6)$ & 17 & & $35.3(12.6-58.0)$ & 16 & & $100.0(100.0-100.0)$ \\
\hline DENV3 & 21 & & $100.0(100.0-100.0)$ & 21 & & $42.9(21.7-64.0)$ & 21 & & $61.9(41.1-82.7)$ & 17 & & $88.2(72.9-103.55)$ \\
\hline DENV4 & 30 & & $100.0(100.0-100.0)$ & 30 & & $86.6(74.5-98.8)$ & 29 & & $82.8(69.0-96.5)$ & 30 & & 86.6 (74.5-98.8) \\
\hline Yellow fever & 19 & & $100.0(100.0-100.0)$ & 19 & & $84.2(67.8-100.6)$ & 14 & & $57.1(31.2-83.1)$ & 16 & & $87.5(71.3-103.7)$ \\
\hline Non-flavivirus & 58 & & $89.7(81.8-97.5)$ & 44 & & 77.2(64.9-89.7) & 8 & & $\begin{array}{l}100.0(100.0- \\
100.0)\end{array}$ & 15 & & $100.0(100.0-100.0)$ \\
\hline Measles \& Rubella & 40 & & $87.5(77.3-97.8)$ & 17 & & $94.1(82.9-105.3)$ & 8 & & $\begin{array}{c}100.0(100.0- \\
100.0)\end{array}$ & 1 & & $100.0(100.0-100.0)$ \\
\hline Hepatitis & 13 & & $92.3(77.8-100.0)$ & 13 & & $69.2(44.2-94.3)$ & - & & & 13 & & $100.0(100.0-100.0)$ \\
\hline Other & 5 & & $100.0(100.0-100.0)$ & 14 & & $64.3(39.2-89.4)$ & - & & & 1 & & $100.0(100.0-100.0)$ \\
\hline $\begin{array}{l}\text { General Population } \\
\text { (Set3) }\end{array}$ & - & & & 52 & & $63.5 \%(48.9-76.4)$ & & & & & & \\
\hline $\begin{array}{l}\begin{array}{l}\text { Overall (all available } \\
\text { samples) }\end{array} \\
\text { s. }\end{array}$ & 333 & 22.2(15.8-28.4) & $96.3(93.5-99.2)$ & $\begin{array}{c}37 \\
2\end{array}$ & 67.8(60.8-74.9) & $74.0(67.4-79.9)$ & 235 & 62.4(53.9-70.9) & $63.6(54.7-72.6)$ & 277 & $31.7(24.7-38.8)$ & 92.7 (87.9-97.6) \\
\hline PPV & & $86.1(72.8-93.4)$ & & & $68.3(62.5-73.5)$ & & & $66.1(59.5-72.1)$ & & & $86.9(76.6-93.1)$ & \\
\hline NPV & & $55.2(53.0-57.3)$ & & & $73.7(68.9-78.0)$ & & & $59.8(53.3-66.0)$ & & & $47.2(44.4-50.1)$ & \\
\hline Accuracy & & $59.2(53.7-64.5)$ & & & 71.2(66.4-75.8) & & & $63.0(56.5-69.2)$ & & & 56.0(49.9-61.9) & \\
\hline Overall ( $\geq 7$ days) & 255 & 32.6(22.9-42.3) & $96.3(93.5-99.2)$ & $\begin{array}{c}29 \\
4\end{array}$ & $86.7(79.6-93.7)$ & 74.0(67.4-79.9) & 167 & $87.7(79.2-96.2)$ & $63.6(54.7-72.6)$ & 199 & 48.3(41.3-55.4) & 92.7 (87.9-97.6) \\
\hline PPV ( $\geq 7$ days) & & $82.9(67.6-91.8)$ & & & $59.5(53.5-65.3)$ & & & $55.6(48.9-62.0)$ & & & $84.3(72.7-91.6)$ & \\
\hline NPV ( $\geq 7$ days) & & $72.7(69.7-75.6)$ & & & $92.6(88.1-95.5)$ & & & $90.9(83.1-95.3)$ & & & $68.3(63.6-72.6)$ & \\
\hline Accuracy ( $\geq 7$ days) & & 74.1(68.3-79.4) & & & $77.9(72.7-82.5)$ & & & 71.9(64.4-78.5) & & & $72.5(65.6-78.6)$ & \\
\hline
\end{tabular}


Table2 - Sensitivity and specificity of the IgM and IgG Euroimmun NS1 commercial ELISA, the MAC-ELISA and IgM $\mu$-capture Novagnost assays with

496 sera from confirmed ZIKV (Set 1) and the Control non-ZIKV group (Set 1 and Set 2). Specificity values were calculated for each assay based on Set 2 and

497 3. Data from ZIKV-positive cases served only for determining the sensitivity and was not used for the specificity calculation. Similarly, data from ZIKV-

498 negative cases serve only for determining the specificity and were not used for calculating the sensitivity. Overall sensitivity, PPV, NPV and accuracy were

499 calculated with both a) all the ZIKV Positive samples and b) only the ZIKV samples collected $\geq 7$ days post symptom onset. Note: Sens., Sensitivity; Spec.,

500 Specificity; CI, Confidence Interval; PPV, Positive Predictive Value; NPV, Negative Predictive Value; ZIKV, Zika virus; DENV, Dengue virus; Days,

501 number of days the sample was collected after symptom onset. Indeterminate results were considered negative for the calculation. Non-flavivirus includes

502 measles \& rubella, hepatitis and general population samples. DENV (all) includes all DENV samples (DENV 1-4). 
Table 3 Sensitivity and specificity for the two anti-DENV antibody ELISAs and the ZIKV PRNT

\begin{tabular}{|c|c|c|c|c|c|c|c|c|c|}
\hline & \multicolumn{3}{|c|}{ IgM DENV Panbio ELISA } & \multicolumn{3}{|c|}{ IgG DENV Panbio ELISA } & \multicolumn{3}{|c|}{ ZIKV PRNT } \\
\hline & Tested (n) & $\%$ Sens. $(95 \% \mathrm{CI})$ & $\%$ Spec. $(95 \%$ CI $)$ & Tested (n) & $\%$ Sens. $(95 \% \mathrm{CI})$ & \% Spec. $(95 \%$ CI) & Tested (n) & \% Sens. $(95 \% \mathrm{CI})$ & \% Spec. $(95 \%$ CI $)$ \\
\hline Zika Positive & 169 & $32.5(25.5-39.6)$ & & 168 & $85.6(80.3-90.9)$ & & 153 & 67.3(59.9-74.8) & \\
\hline Zika ( 1-6 Days) & 79 & $22.8(15.7-29.8)$ & & 78 & $83.3(75.1-91.6)$ & & 65 & $35.4(23.8-47.0)$ & \\
\hline Zika ( $\geq 7-13$ Days) & 41 & $51.2(44.2-58.3)$ & & 41 & $85.4(74.5-96.2)$ & & 40 & $87.5(77.3-97.7)$ & \\
\hline Zika ( $\geq 14$ Days) & 49 & $32.7(25.6-39.7)$ & & 49 & $89.8(81.3-98.3)$ & & 48 & 93.8(86.9-100.0) & \\
\hline Zika ( $\geq 7$ Days) & 90 & 41.1(34.0-48.2) & & 90 & $87.8(81.0-94.5)$ & & 88 & $90.9(84.9-96.9)$ & \\
\hline Non ZIKV (Flavivirus and Non-flavivirus) & 159 & & $42.1(34.5-49.8)$ & 135 & & $28.2(20.6-35.7)$ & 102 & & $96.1(96.0-96.1)$ \\
\hline DENV (all) & 90 & & $16.7(9.0-24.4)$ & 88 & & $18.2(10.1-26.2)$ & 71 & & $94.4(94.3-94.4)$ \\
\hline DENV1 & 21 & & $4.8(0.0-13.9)$ & 21 & & 47.6(26.3-67.0) & 14 & & $100(100-100)$ \\
\hline DENV2 & 17 & & $0(0-0)$ & 16 & & $0(0-0)$ & 12 & & $100(100-100)$ \\
\hline DENV3 & 21 & & $4.76(0-13.9)$ & 20 & & $15.0(0.0-30.65)$ & 16 & & $75.0(74.8-75.2)$ \\
\hline DENV4 & 31 & & $41.94(24.6-59.3)$ & 31 & & $9.7(0.0-20.1)$ & 29 & & $100(100-100)$ \\
\hline Yellow fever & 19 & & $84.21(67.8-100.6)$ & 18 & & $50.0(26.9-73.1)$ & 15 & & $100(100-100)$ \\
\hline Non-flavivirus & 50 & & $72.0(59.6-84.5)$ & 29 & & $44.8(26.7-62.9)$ & 15 & & $100(100-100)$ \\
\hline Measles \& Rubella & 29 & & $65.5(48.2-82.8)$ & 12 & & $91.7(76.0-107.3)$ & & & \\
\hline Hepatitis & 12 & & $66.7(40.0-93.3)$ & 13 & & $15.4(0.0-35.0)$ & 16 & & $100(100-100)$ \\
\hline Other & 9 & & $100(100-100)$ & 4 & & $0(0-0)$ & & & \\
\hline Overall (all available samples) & 328 & $32.5(25.5-39.6)$ & $42.1(34.5-49.8)$ & 303 & $85.6(80.3-90.9)$ & $28.2(20.6-35.7)$ & 256 & $67.3(59.9-74.8)$ & $96.0(96.0-96.1)$ \\
\hline PPV & & $37.4(31.7-43.5)$ & & & $59.6(56.6-62.5)$ & & & $96.3(90.7-98.5)$ & \\
\hline NPV & & $37.0(32.3-42)$ & & & $61.3(50.0-71.5)$ & & & $66.2(60.9-71.2)$ & \\
\hline Accuracy & & $37.2(32.0-42.7)$ & & & $59.9(54.2-65.5)$ & & & 78.8(73.3-83.7) & \\
\hline Overall ( $\geq 7$ days) & 249 & 41.1(34-48.2) & $42.1(34.5-49.8)$ & 225 & 87.8(82.5-93.1) & $28.2(20.6-35.7)$ & 191 & $90.9(84.9-96.9$ & $96.1(96.0-96.1)$ \\
\hline PPV ( $\geq 7$ days) & & $28.7(20.9-36.5)$ & & & $44.9(37.5-52.2)$ & & & $95.2(88.4-98.1)$ & \\
\hline NPV ( $\geq 7$ days) & & $61.9(53.8-69.9)$ & & & 76.6(64.5-88.7) & & & $92.4(86.3-96.0)$ & \\
\hline Accuracy ( $\geq 7$ days) & & 45.9(39.9-51.9) & & & $51.6(45.0-58.1)$ & & & $93.7(89.2-96.7)$ & \\
\hline
\end{tabular}


510 Table 3 - The sensitivity and specificity of the IgM and IgG Panbio DENV commercial ELISA and ZIKV PRNT with the ZIKV panel (Set 1)

511 and the Control non-ZIKV group (Set 2). Specificity values were calculated for each assay based on Set 2. Data from ZIKV-positive cases

512 served only for determining the sensitivity and was not used for the specificity calculation. Similarly, data from ZIKV-negative cases serve only

513 for determining the specificity and were not used for calculating the sensitivity. Overall sensitivity, PPV, NPV and accuracy were calculated

514 with ZIKV Positive samples collected $\geq 7$ days post symptom onset.

515 Note: Sens., Sensitivity; Spec., Specificity; CI, Coefficient Interval; PPV, Positive Predictive Value; NPV, Negative Predictive Value; ZIKV,

516 Zika virus; DENV, Dengue virus; Days, number of days the sample was collected after symptom onset. Indeterminate results were considered

517 negative for the calculation. Non-flavivirus includes measles \& rubella, hepatitis and general population samples. DENV (all) includes all

518 DENV samples (DENV 1-4). 


\section{$519 \quad$ Figure Legends}

521 Figure 1 Zika antibody detected in serum samples collected during the acute (1-6 days after

522 onset), early-convalescent phase (7-13d) and late convalescent-phase ( $\geq 14 d)$ from PCR positive

523 Zika cases measured by IgM (A) or IgG (B) NS1 anti-ZIKV ELISAs (Euroimmun). C) IgM NS1

524 anti-ZIKV ELISA measurements for acute (1-6 days after onset) and convalescent ( $\geq 7$ days)

525 samples from PCR positive ZIKV cases. D) IgG NS1 anti-ZIKV antibody levels in paired serum

526 samples from PCR positive ZIKV positive cases. Dotted horizontal lines represent the cut-off

527 value used in each assay. Data points above the cut-off are considered positive. Trend-line in C)

528 and D) represent the median antibody levels for acute and convalescent samples. Statistically

529 significant differences between two groups were measured by Mann Whitney U test $(* * *$

$530 \mathrm{p}=0.0001)$. Figure shows antibody Ratios* calculated as per manufacturers' instructions

531 (Antibody Ratio = OD Sample/OD Calibrator).

Figure 2 The change in IgG NS1 Euroimmun anti-ZIKV antibody levels between paired serum

534 samples from PCR positive Zika cases by day of collection (days post symptom onset) of the

535 first (acute) sample. Based on the first sample (collected 0 -7 days) and second sample (median

536 interval between samples was 7 days). The highest fold change (change in antibody level

537 between $1^{\text {st }}$ and $2^{\text {nd }}$ samples) was observed among paired samples collected on days 2 and 7 post

538 symptom onset.

540 Figure 3 Anti-ZIKV antibody levels in sequential serum samples collected from Zika PCR

541 positive cases on different days post symptom onset (0-54 days) measured in A) IgM 
542 Euroimmun NS1 and B) IgG Euroimmun NS1 anti-ZIKV ELISAs. Dotted line shows assay cut-

543 offs. The figure shows more consistent detection of anti-Zika antibodies (level above the cut-off)

544 among convalescent samples when measuring IgG compared to IgM. Ratios calculated as per

545 manufacturers' instruction; 1st collection (acute sample [closed circles]); 2nd collection

546 (convalescent samples [open squares]); 3rd collection (late convalescent samples [closed

547 triangles])

549 Figure 4 Correlation between anti-ZIKV and anti-DENV antibody levels in individual sera

550 samples. A) IgG anti-DENV ELISA (Panbio) versus IgG anti-ZIKV NS1 (Euroimmun) ELISA.

551 Anti-DENV and anti-ZIKV IgG antibody levels showed a positive correlation. When a patient

552 exhibited a relatively high anti-DENV IgG antibody response they also tended to exhibit a

553 relatively high anti-ZIKV IgG antibody response ( $\mathrm{p}<0.001 ; \mathrm{r} 2=0.258 ; \mathrm{n}=168) ; \mathrm{B}) \operatorname{IgM}$ DENV

554 ELISA and IgM ZIKV NS1 ELISA antibody levels. Again the measurements showed a positive

555 correlation $(\mathrm{p}=0.015 ; \mathrm{r} 2=0.015 ; \mathrm{n}=166)$. Dotted lines show assay cut-offs. Dashed line shows the

556 best-fitting line (Spearman rank correlation [r2]). Correlation was more significant between

557 anti-ZIKV and anti-DENV IgG antibody measurements. The correlation in antibody

558 measurement between ZIKV and DENV ELISAs suggests a degree of overlap in patient

559 responses and/or cross-reaction in antibody detection.

561 Figure 5. The change in anti-ZIKV IgM antibody levels by day post symptom onset in

562 sequential sera from ZIKV PCR positive cases $(n=4)$. Plots A-D represent 4 different patients.

563 Each patient had at least five sequential sera samples collected. Anti-ZIKV IgM was measured 
564 by NS1 (Euroimmun; shown as squares) and $\mu$-capture N (Novagnost; shown as circles)

565 ELISAs. Dotted lines represent cut-off values for each assay. Ratios calculated as recommended

566 by the manufacturers. The plots display a unique pattern of ZIKV IgM antibody response over

567 time for each patient.

569 Figure 6 Receiver Operating Characteristic (ROC) curve comparing sensitivity and specificity at

570 different cut-off values for the anti-ZIKV IgG NS1 ELISA (n=294 sera); A) ROC Curve; B)

571 Specificity and Sensitivity at each cut-off. The dotted line in B indicates the cut-off

572 recommended by the manufacturer (ratio of 1.1). The accuracy of IgG NS1 ELISA was 77.9\%

573 using the manufacturer's cut-off. Higher accuracy was observed when the cut-off was increased

574 to 1.5 (where the curves intersect on plot B). Using this cut-off, the ELISA had an accuracy of

$57581.0 \%$. Sensitivity and specificity were 78.9 and $82.2 \%$ respectively.

577 Figure 7 Diagram representing the different patterns of anti-viral IgG and IgM antibody

578 responses and viral RNA detection observed in sera over days from symptom onset among (A)

579 virus naïve and (B) previously exposed individuals. The cartoon exhibits a more prominent IgG

580 response compared to IgM among individuals previously exposed to the virus. In our current

581 study, we observed a more prominent anti-ZIKV IgG compared to IgM response (see Figure 3). 
bioRxiv preprint doi: $h t$ Ss://doi.org/10.1101/2020.11.25.399386; this version posted November 27, 2020. The copyright holder for this preprint (which was not certified by peer review) is the author/funder, who has granted bioRxiv a license to display the preprint in perpetuity. It is made
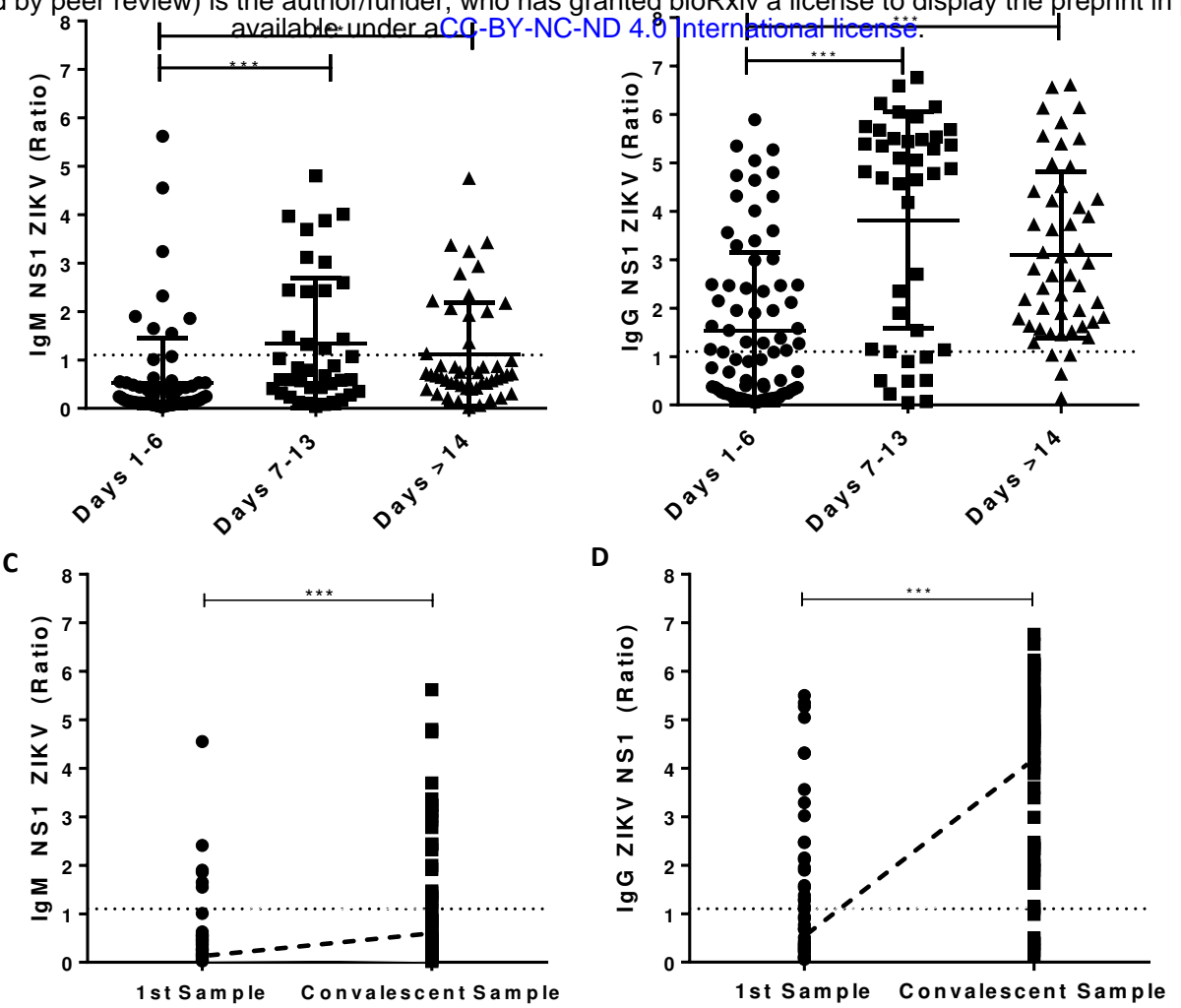

Figure 1 Zika antibody detected in serum samples collected during the acute (1-6 days after onset), earlyconvalescent phase (7-13d) and late convalescent-phase ( $\geq 14 d)$ from PCR positive Zika cases measured by IgM (A) or IgG (B) NS1 anti-ZIKV ELISAs (Euroimmun). C) IgM NS1 anti-ZIKV ELISA measurements for acute (1-6 days after onset) and convalescent ( $\geq 7$ days) samples from PCR positive ZIKV cases. D) IgG NS1 anti-ZIKV antibody levels in paired serum samples from PCR positive ZIKV positive cases. Dotted horizontal lines represent the cut-off value used in each assay. Data points above the cut-off are considered positive. Trend-line in C) and D) represent the median antibody levels for acute and convalescent samples. Statistically significant differences between two groups were measured by Mann Whitney $U$ test $(* * * p=0.0001)$. Figure shows antibody Ratios* calculated as per manufacturers' instructions (Antibody Ratio = OD Sample/OD Calibrator). 


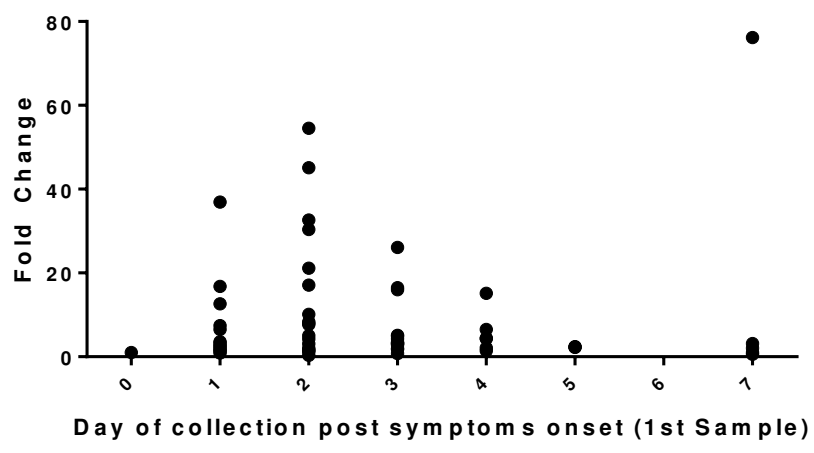

Figure 2 The change in IgG NS1 Euroimmun anti-ZIKV antibody levels between paired serum samples from PCR positive Zika cases by day of collection (days post symptom onset) of the first (acute) sample. Based on the first sample (collected $0-7$ days) and second sample (median interval between samples was 7 days). The highest fold change (change in antibody level between $1^{\text {st }}$ and $2^{\text {nd }}$ samples) was observed among paired samples collected on days 2 and 7 post symptom onset. 


\section{A}

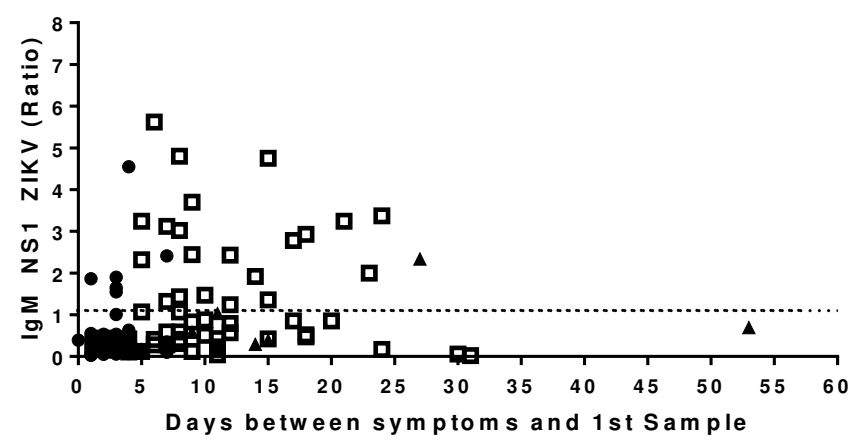

B

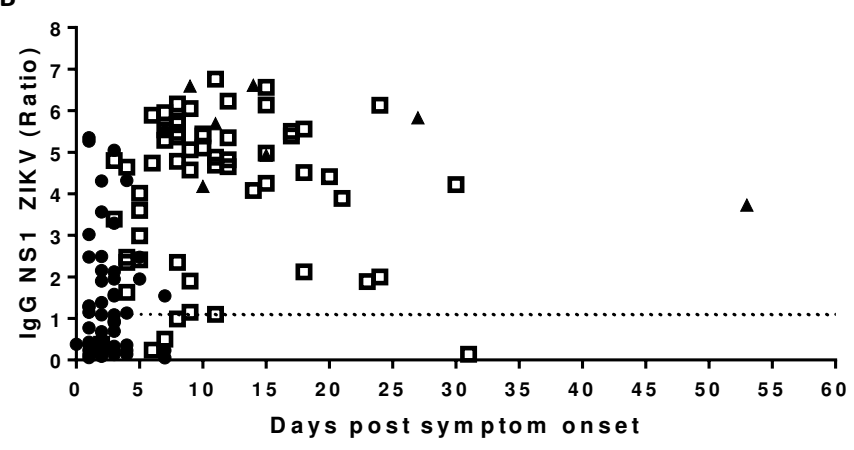

Figure 3 Anti-ZIKV antibody levels in sequential serum samples collected from Zika PCR positive cases on different days post symptom onset (0-54 days) measured in A) IgM Euroimmun NS1 and B) IgG Euroimmun NS1 anti-ZIKV ELISAs. Dotted line shows assay cut-offs. The figure shows more consistent detection of antiZika antibody (level above the cut-off) among convalescent samples when measuring IgG compared to IgM. Ratios calculated as per manufacturers' instruction; 1st collection (acute sample [closed circles]); 2nd collection (convalescent samples [open squares]); 3rd collection (late convalescent samples [closed triangles]) 
A

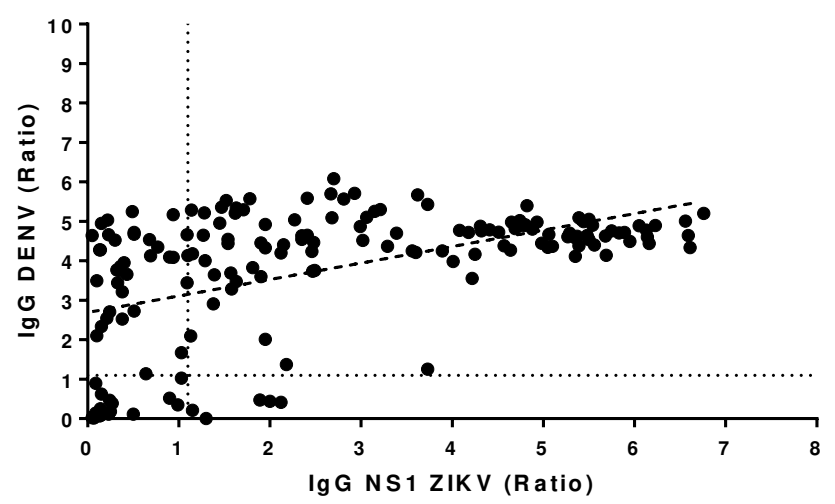

B

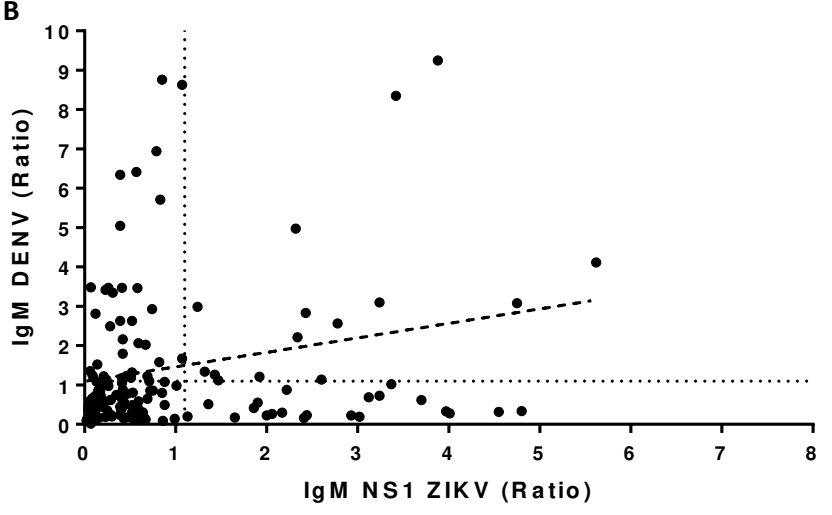

Figure 4 Correlation between anti-ZIKV and anti-DENV antibody levels in individual sera samples. A) IgG antiDENV ELISA (Panbio) versus IgG anti-ZIKV NS1 (Euroimmun) ELISA. Anti-DENV and anti-ZIKV IgG antibody levels showed a positive correlation. When a patient exhibited a relatively high anti-DENV IgG antibody response they also tended to exhibit a relatively high anti-ZIKV IgG antibody response $(p<0.001 ; r 2=0.258$; n=168); B) IgM DENV ELISA and IgM ZIKV NS1 ELISA antibody levels. Again the measurements showed a positive correlation $(p=0.015 ; r 2=0.015 ; n=166)$. Dotted lines show assay cut-offs. Dashed line shows the bestfitting line (Spearman rank correlation [r2]). Correlation was more significant between anti-ZIKV and antiDENV IgG antibody measurements. The correlation in antibody measurement between ZIKV and DENV ELISAS suggests a degree of overlap in patient responses and/or cross-reaction in antibody detection. 


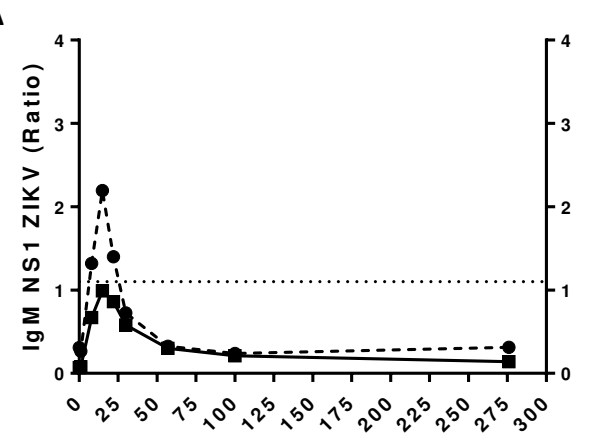

C

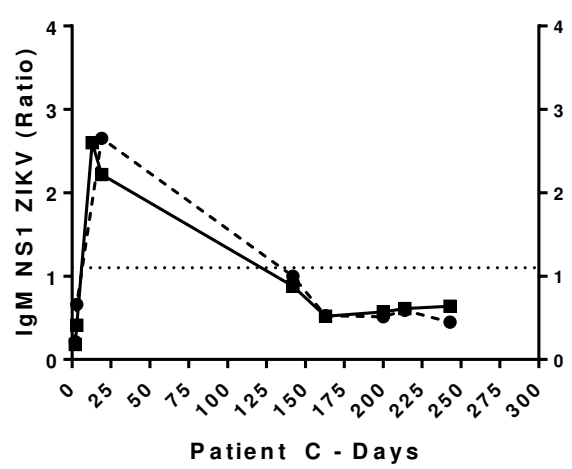

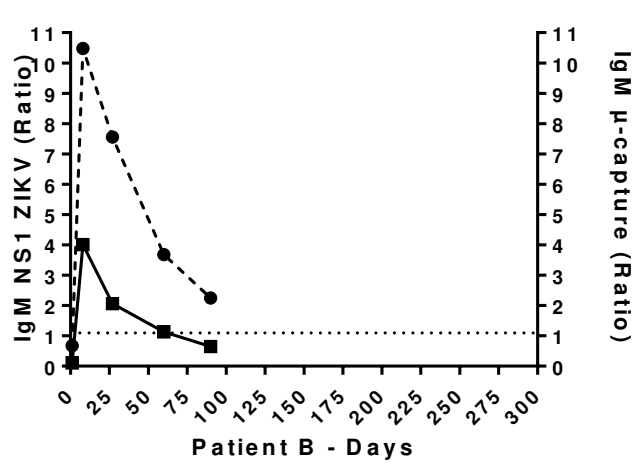

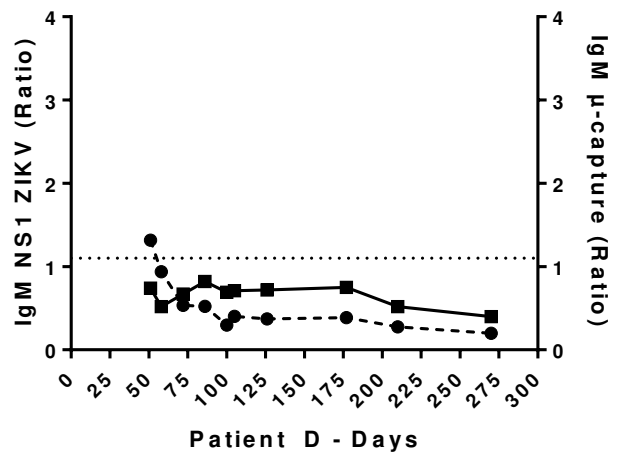

Figure 5. The change in anti-ZIKV IgM antibody levels by day post symptom onset in sequential sera from ZIKV PCR positive cases $(n=4)$. Plots A-D represent 4 different patients. Each patient had at least five sequential sera samples collected. Anti-ZIKV IgM was measured by NS1 (Euroimmun; shown as squares) and $\mu$-capture $N$ (Novagnost; shown as circles) ELISAs. Dotted lines represent cut-off values for each assay. Ratios calculated as recommended by the manufacturers. The plots display a unique pattern of ZIKV IgM antibody response over time for each patient. 
bioRxiv preprint doi: https://doi.org/10.1101/2020.11.25.399386; this version posted November 27, 2020. The copyright holder for this preprint (which was not certified by peer review) is the author/funder, who has granted bioRxiv a license to display the preprint in perpetuity. It is made available under aCC-BY-NC-ND 4.0 International license.

A

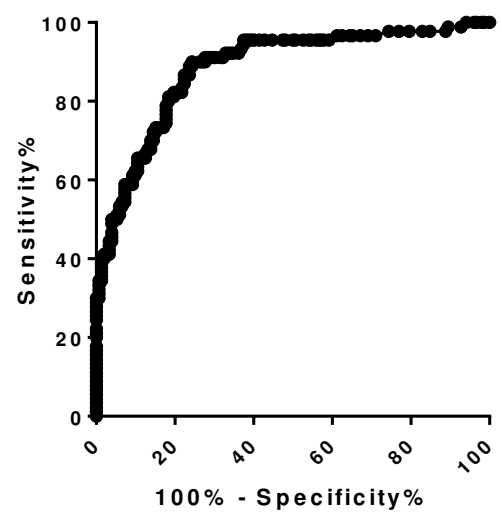

B

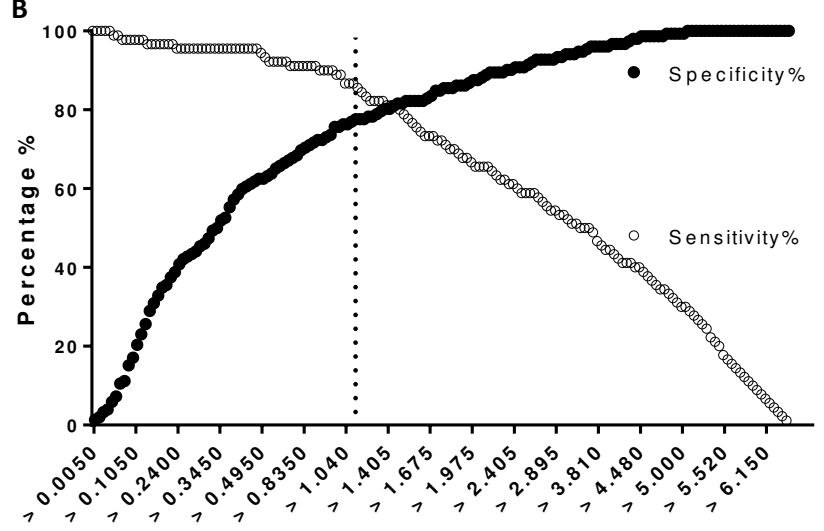

IgG NS1 Cut-off values

Figure 6 Receiver Operating Characteristic (ROC) curve comparing sensitivity and specificity at different cut-off values for the anti-ZIKV IgG NS1 ELISA (n=294 sera); A) ROC Curve; B) Specificity and Sensitivity at each cut-off. The dotted line in B indicates the cut-off recommended by the manufacturer (ratio of 1.1). The accuracy of IgG NS1 ELISA was $77.9 \%$ using the manufacturer's cut-off. Higher accuracy was observed when the cut-off was increased to 1.5 (where the curves intersect on plot B). Using this cut-off, the ELISA had an accuracy of $81.0 \%$. Sensitivity and specificity were 78.9 and $82.2 \%$ respectively. 

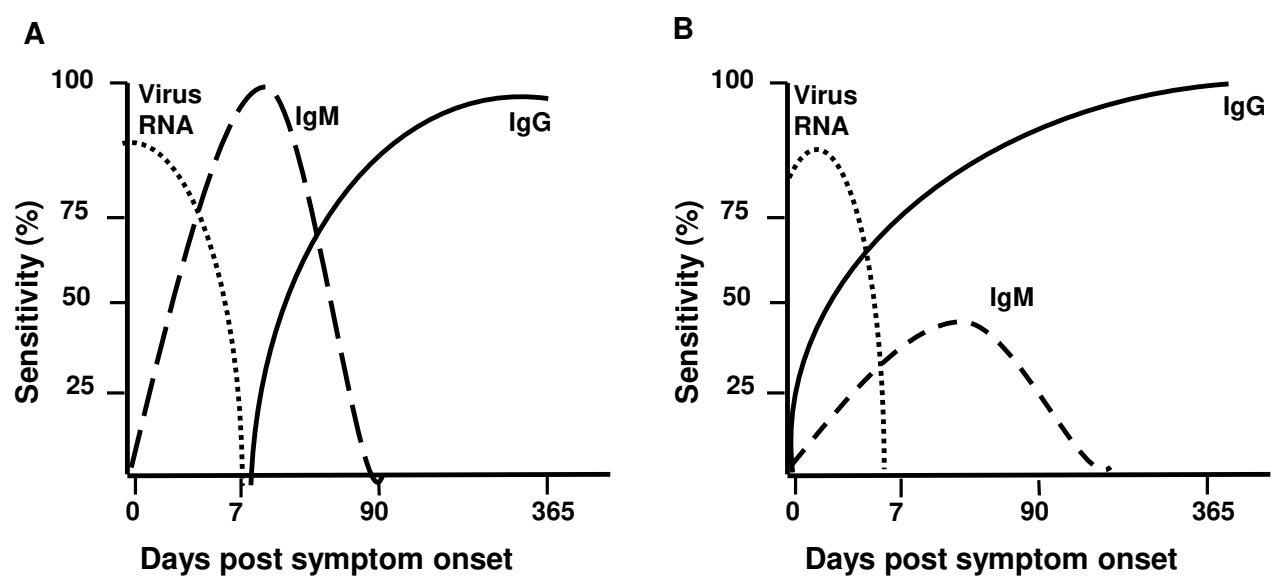

Figure 7 Diagram representing the different patterns of anti-viral IgG and IgM antibody responses and viral RNA detection observed in sera over days from symptom onset among (A) virus naïve and (B) previously exposed individuals. The cartoon exhibits a more prominent IgG response compared to IgM among individuals previously exposed to the virus. In our current study, we observed a more prominent anti-ZIKV IgG compared to IgM response (see Figure 3). 